Artículo Original

\title{
ROCURONIO EN OBESOS: ¿DEBE DOSIFICARSE POR PESO REAL O PESO IDEAL?
}

\author{
Dagoberto Ojeda D. ${ }^{1}$, Florence Gazabatt S. ${ }^{1}$, Ricardo Bustamante B. ${ }^{1}$, \\ Álvaro Burgos W. ${ }^{2}$ y Patricia Cisternas M. ${ }^{1}$
}

Key words: Rocuronium bromide, obesity, administration and dosage.

\begin{abstract}
Resumen
El aumento universal en la prevalencia de obesidad ha causado que los anestesiólogos se vean frecuentemente enfrentados a anestesiar pacientes obesos. Pese a esto aún existen dudas respecto a cómo dosificar las drogas en estos pacientes. La literatura recomienda dosificar el rocuronio en obesos en base al peso ideal (IBW, iniciales del inglés Ideal Body Weight) pero esta sugerencia está basada más bien en la prudencia que en la evidencia. Se decidió explorar con análisis de sobrevida (análisis tiempo-evento), la duración del rocuronio en obesos al ser dosificado por peso ideal (IBW) y por peso real (TBW, iniciales del inglés de peso corporal total). Al administrar el rocuronio en base al peso real (TBW) se observó una prolongación en su duración de acción con un acortamiento de su latencia en relación con la dosificación en base al peso ideal (IBW). Hubo una marcada prolongación de la duración de acción y el índice de recuperación tanto al dosificar por peso real como peso ideal. Debido a esta sensibilidad aumentada al rocuronio en pacientes obesos recomendamos dosificarlo en base al peso ideal, excepto si se necesita intubar rápidamente.
\end{abstract}

\begin{abstract}
The universal increase in prevalence of obesity has caused that anesthesiologist are frequently encountered with anesthetizing obese patients. There stills exists doubt on how to dosage drugs to these patients. Literature recommends dosing rocuronium in the obese based on ideal weight (IBW), being this suggestion based more on prudence than on evidence. Randomized control trial was designed for obese patients scheduled for bariatric surgery to study the pharmacodynamics of rocuronium in this population. Patients were randomly assigned into two groups: rocuronium dosage $0.6 \mathrm{mg} \cdot \mathrm{kg}^{-1}$ for real weight (TBW) or rocuronium dosage of 0.6 $\mathrm{mg} \cdot \mathrm{kg}^{-1}$ for ideal weight (IBW). Ideal weight was calculated according to Lemmens formula. The reference group was the dosage based on IBW. Previous calibration, evaluating the first twitch of train-of-four $\left(\mathrm{T}_{1}\right)$, we registered onset time, clinical duration, recovery index, level of muscle relaxation to which the first reinforcement was administered and clinical duration of rocuronium reinforcement. A total of 100 patients were part of the study: 54 in group TBW and 46 in group IBW. When administering rocuronium based on real weight (TBW) a prolongation in clinical duration was found: Medium (IQR*); 79.5 (67 - 105) minutes versus 44.5 (33 - 63.5 minutes) in the IBW group $(\mathrm{p}<0.001)$ with a decrease in latency in the TBW group $120(90-150$ secs.) versus the IBW group $180(120-270$ secs. $)(\mathrm{p}<0.001)$. There were no differences in the recovery index between both levels of dosing. There was a marked prolongation of both clinical duration and recovery index in both groups, as supported in some literature. Based on the evidence, we recommend to dose rocuronium in obese patients based on ideal weight, with the exception of cases were quick intubation is required.

* IQR = interquartile range.
\end{abstract}

\footnotetext{
Médico Anestesiólogo Clínica Dávila.

2 Becado Anestesiología Universidad de Los Andes.
} 


\section{INTRODUCCIÓN}

La obesidad, definida como un Índice de Masa Corporal $\geq 30 \mathrm{~kg} \cdot \mathrm{m}^{-2}$ es una epidemia global; la prevalencia de obesidad en USA se estima en más de $35 \%^{1,2}$. En Chile, la prevalencia de obesidad aumentó entre el año 2003 y el 2010 de $23,2 \%$ a $25,1 \%{ }^{3}$. La cirugía bariátrica se considera una alternativa terapéutica en caso de que el IMC sea mayor de $40 \mathrm{~kg} \cdot \mathrm{m}^{-2}$ o cuando el IMC está entre 35 y 40 $\mathrm{kg} \cdot \mathrm{m}^{-2}$ pero asociado a otras comorbilidades ${ }^{4}$. Un centro nacional comunicó un aumento de los casos anuales de cirugía bariátrica de 100 a 700 casos entre el año 2000 y el $2008^{5}$. Pareciera entonces que es necesario anestesiar cada vez más frecuentemente pacientes obesos, tanto para cirugía bariátrica como para otros procedimientos.

Pese a este aumento mundial en la prevalencia de obesidad, no existe consenso acerca de cómo deben ser ajustadas las dosis de los medicamentos en los pacientes obesos ${ }^{6}$. La obesidad modifica la farmacocinética y la farmacodinamia de las drogas anestésicas debido a un aumento del gasto cardiaco, de la masa magra, del tejido graso y del volumen extracelular?.

La intubación traqueal debe ser rápida en pacientes obesos puesto que, en condiciones de apnea, la desaturación de la hemoglobina es más acelerada que en pacientes de peso normal debido a una disminución de la capacidad residual funcional y un aumento del consumo de $\mathrm{O}_{2}{ }^{8-10}$. Además, la obesidad frecuentemente se asocia a apnea del sueño ${ }^{11} \mathrm{y}$ ambas condiciones son factores de riesgo para dificultad en el manejo de la vía aérea con máscara facial ${ }^{12,13}$. Por estos motivos tradicionalmente se ha recomendado a la succinilcolina como relajante muscular de elección para la intubación traqueal en obesos ${ }^{14}$.

El rocuronio es un relajante muscular de tipo aminoesteroidal que contiene un grupo amonio cuaternario altamente ionizado a $\mathrm{pH}$ fisiológico por lo que es una droga hidrofílica, con poca afinidad por el tejido graso y que se distribuye en el volumen extracelular. Tiene un rápido inicio de acción, cercano al de la succinilcolina pero sin los efectos secundarios asociados a esta última ${ }^{15}$, esto lo hace ser un relajante muscular apropiado para ser utilizado en pacientes obesos.

Se ha sugerido que las dosis de rocuronio en obesos sean calculadas en base al peso ideal ${ }^{7,8,11,14,16}$ pero esta recomendación está basada más bien en la prudencia que en la evidencia científica ${ }^{17}$. Por esta razón, se decidió diseñar un estudio con el objetivo de comparar el comportamiento del rocuronio al administrarlo en base a peso real (TBW) y a peso ideal (IBW) en pacientes obesos.

\section{MATERIAL Y MÉTODOS}

Previa aprobación del comité de ética de nuestra institución, fueron enrolados en el estudio pacientes obesos que serían operados de cirugía bariátrica. Se solicitó el consentimiento informado de los pacientes. Se excluyeron aquellos aquejados de enfermedad hepática, (a excepción de hígado graso), renal y neuromuscular.

Se estudiaron 100 pacientes que fueron asignados aleatoriamente a 2 grupos:

- Grupo 1: Uso de rocuronio $0,6 \mathrm{mg} \cdot \mathrm{kg}^{-1}$ en base a peso real.

- Grupo 2: Uso de rocuronio $0,6 \mathrm{mg} \cdot \mathrm{kg}^{-1}$ en base a peso ideal.

El peso ideal fue calculado en base a la fórmula propuesta por Lemmens ${ }^{19}$ :

\section{Peso ideal $=22 \times(\text { Estatura en } \mathrm{m})^{2}$}

Esta fórmula fue elegida puesto que entrega valores intermedios entre todas las fórmulas publicadas en la literatura para calcular el peso ideal.

La monitorización neuromuscular se efectuó con acelerografía, (TOF-Watch ${ }^{\circledR}$ SX, Organon), que permite almacenar computacionalmente la información electromiográfica. Se instalaron los electrodos en el borde cubital del antebrazo, se instaló el dispositivo de precarga del pulgar y el resto de los dedos se inmovilizaron fijándolos al apoya-brazo de la mesa quirúrgica. Se utilizó la primera respuesta del TOF $\left(\mathrm{T}_{1}\right)$ para la evaluación del bloqueo neuromuscular.

La mesa operatoria se dispuso en posición de elevación del tórax en $25^{\circ}$, sin cojín de rampa. Previa preoxigenación por 5 minutos se realizó la inducción de la anestesia con fentanyl y propofol en dosis suficientes para perder el reflejo palpebral. Posteriormente se realizó la calibración del estímulo supramáximo del aceleromiógrafo, tras lo cual se administró el rocuronio según la dosis determinada para cada grupo (Esmeron ${ }^{\circledR}$ Organon, Oss, Holanda) a temperatura ambiental y se inició la monitorización del tren de cuatro estímulos (TOF) cada 15 segundos durante toda la cirugía. Se consideró como tiempo cero al final de la inyección de rocuronio. La intubación traqueal se realizó una vez alcanzado el máximo de supresión de $T_{1}$. Nada puesto que ese análisis finalmente no fue utilizado. Se consignó la $\mathrm{SaO}_{2}$ inmediatamente después de la intubación traqueal.

El anestesista a cargo fue ciego al grupo asignado a su paciente, pero tuvo acceso a la información cuantitativa del estimulador de nervio periférico, 
de modo de poder administrar las dosis de refuerzo que considerara necesaria. La mantención de la anestesia se realizó con sevoflurano o desflurano en una mezcla de $\mathrm{O}_{2}$ /aire. Al final de la cirugía se revirtió el bloqueo residual con neostigmina o sugammadex para alcanzar una relación $\mathrm{T}_{4} / \mathrm{T}_{1} \geq 1$.

De los datos registrados se analizaron: el tiempo de inicio de acción (tiempo transcurrido desde el final de la inyección del rocuronio hasta la máxima depresión de $\mathrm{T}_{1}$ ), la duración clínica (tiempo transcurrido hasta el $25 \%$ de $\mathrm{T}_{1}$ ), el tiempo hasta la recuperación espontánea al $75 \%$ y al $90 \%$ de $\mathrm{T}_{1}$, y el índice de recuperación 25-75 (intervalo de tiempo transcurrido entre el $25 \%$ y el $75 \%$ de la recuperación de $\mathrm{T}_{1}$ ). En los casos en los que se usó refuerzos de rocuronio, se registró el porcentaje de $\mathrm{T}_{1}$ al que se administró el refuerzo y el tiempo hasta recuperar un $25 \%$ of $\mathrm{T}_{1}$ después del primer refuerzo. El análisis estadístico se realizó por intención de tratar; para analizar los datos de tiempo se utilizó Log-rank test ${ }^{20,21}$.

Los análisis estadísticos se realizaron en STATA software (STATA versión 10.0, College Station, Tx, USA) y los gráficos en GraphPad PRISMR versión 5.0 para Mac (GraphPad software, San Diego California, USA). El tamaño muestral estimado fue de 96 pacientes y se calculó en STATA en base a STpower $^{18}$ (Survival time power), considerando una duración de acción del doble de tiempo cuando se dosifica por peso real en relación a peso ideal, con un error tipo I de $5 \%$ y una potencia de $90 \%$.

\section{RESULTADOS}

La muestra estudiada estaba caracterizada por un predominio femenino, clasificación de ASA II, IMC mayores de $35 \mathrm{Kg} \cdot \mathrm{m}^{-2}$, alta tasa de hígado graso (mayor de $80 \%$ ). La mayor parte de los pacientes fueron sometidos a una manga gástrica y se usó predominantemente sevoflurano como halogenado (Tabla 1). Los grupos en estudio fueron absolutamente comparables.

\section{Tiempo de inicio de acción}

El tiempo de inicio de acción fue significativamente más prolongado en el grupo dosificado por peso ideal; mediana; (IQR): $180 \mathrm{~s}$ (120-270) versus el grupo dosificado por peso real; $120 \mathrm{~s}(90-150)$ (Figura 1).

Dos de los pacientes del TBW (4\%), no alcanzaron un $100 \%$ de depresión del estímulo único (primera respuesta al TOF) versus 4 pacientes (9\%) del grupo IBW, pero esta diferencia no fue significativa $(p=0,410)$. Es de notar que pese a la prolon-

Tabla 1. Características demográficas y otras

\begin{tabular}{|c|c|c|c|}
\hline & $\begin{array}{c}\text { Dosis TBW } \\
\mathbf{n}=\mathbf{5 4}\end{array}$ & $\begin{array}{c}\text { Dosis IBW } \\
n=46\end{array}$ & $\mathbf{p}$ \\
\hline Mujeres (\%) & 70 & 63 & 0,4370 \\
\hline Edad (años)* & $37,8 \pm 11,8$ & $36,2 \pm 9,0$ & 0,4286 \\
\hline $\operatorname{IMC}\left(\mathrm{kg} \cdot \mathrm{m}^{-2}\right)$ & $38,2(31-50)$ & $39,1(32-56)$ & 0,5000 \\
\hline Clasificación ASA II/III (\%) & $90 / 10$ & $85 / 15$ & 0,3990 \\
\hline Hígado Graso (\%) & 92 & 84 & 0,2230 \\
\hline Manga /Bypass Gástrico (\%) & $74 / 26$ & $85 / 15$ & 0,1900 \\
\hline Fentanyl $\left(\mu \mathrm{g} \cdot \mathrm{kg}^{-1}\right)^{* *}$ & $3,6(2,2-7,8)$ & $4,0(2,5-9,0)$ & 0,4073 \\
\hline Propofol $\left(\mathrm{mg} \cdot \mathrm{kg}^{-1}\right)^{*}$ & $1,9 \pm 0,3$ & $2,0 \pm 0,4$ & 0,1780 \\
\hline$\%$ Sevoflurano/Desflurano & $80 / 20$ & $68 / 32$ & 0,2140 \\
\hline Tiempo Qx (min)** & $109(53-240)$ & $95(62-250)$ & 0,1456 \\
\hline$\%$ Refuerzo de rocuronio & 24 & 41 & 0,0660 \\
\hline$\%$ Altura de $\mathrm{T}_{1}$ en el refuerzo & $50 \pm 33,3$ & $38 \pm 24,7$ & 0,2466 \\
\hline Dosis de refuerzo $\left(\mathrm{mg} \bullet \mathrm{kg}^{-1}\right)$ & $0,14 \pm 0,06$ & $0,14 \pm 0,04$ & 0,8766 \\
\hline $\mathrm{N}^{\circ}$ de refuerzos $1 / 2 / 3(\%)^{* * *}$ & $85 / 15 / 0$ & $74 / 21 / 5$ & 0,6270 \\
\hline$\%$ Neostigmina/Sugammadex & $96 / 4$ & $100 / 0$ & 0,5020 \\
\hline
\end{tabular}

TBW: por peso real; IBW: por peso ideal; *Media \pm SD; **Mediana; min.-máx.; ***13 pacientes requirieron 1 o más refuerzos en el grupo TBW y 19 en IBW (porcentajes calculados sobre esos valores). 


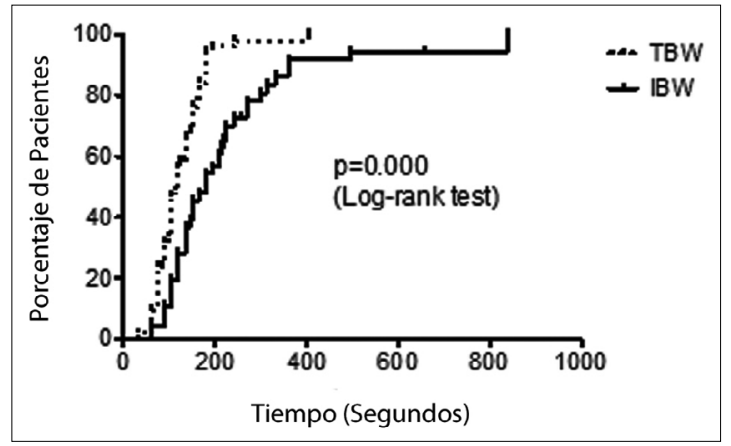

Figura 1. Tiempos de inicio de acción en relación a tipo de dosis utilizada.

gación del tiempo de inicio no hubo diferencias en la saturación de oxígeno inmediatamente después de intubar entre ambos grupos de dosis (Figura 2), 2 pacientes tuvieron saturaciones de oxígeno menores a $90 \%(88 \%)$, ambos pertenecientes al grupo peso real (TBW).

\section{Duración clínica}

El tiempo hasta recuperar $25 \%$ de $T_{1}$ fue influenciado significativamente por la dosis; Mediana (IQR): 79,5 (67-105) minutos en los pacientes dosificados en base al peso real versus 44,5 (33-63,5) minutos en los que se usó dosis basada en el peso ideal (Figura 3). En la Tabla 2, se presenta un resumen de los tiempos de duración del rocuronio para los distintos grupos de pacientes.

\section{Índice de recuperación}

El índice de recuperación fue más prolongado en los pacientes que recibieron dosis de rocuronio por peso real. Sin embargo, esta diferencia no fue significativa: $35 \%$ de los pacientes del grupo TBW no alcanzaron un $75 \%$ de recuperación versus un

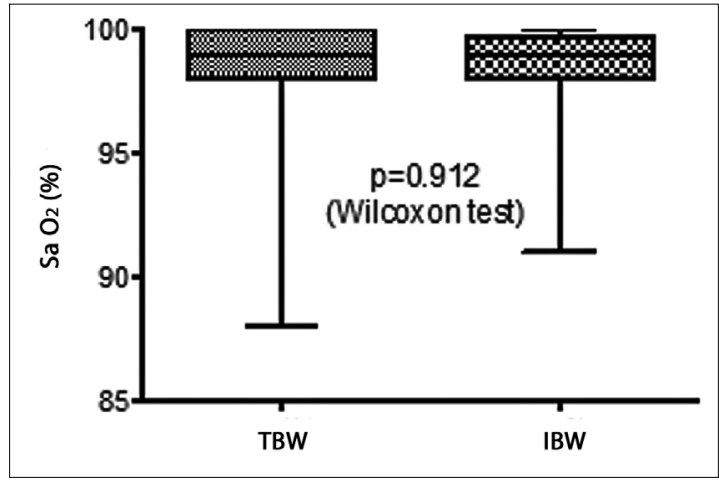

Figura 2. Saturación de oxígeno después de la intubación según el tipo de dosis utilizada.

$33 \%$ en el grupo IBW $(\mathrm{p}=0,786)$. Estos pacientes fueron incorporados al análisis como right censoring data, vale decir, como pacientes que no alcanzaron el objetivo señalado.

\section{Dosis de refuerzo}

$41,3 \%$ de los pacientes dosificados por IBW requirieron refuerzo de rocuronio versus $24 \%$ en los

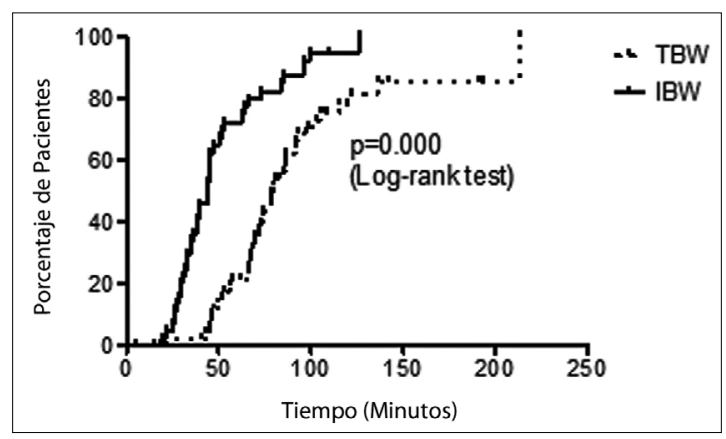

Figura 3. Duración clínica según el tipo de dosis utilizada.

Tabla 2. Latencia y duración del rocuronio en obesos según dosis; Mediana (IQR)

\begin{tabular}{|lccc|}
\hline & TBW & IBW & p \\
Tiempo de inicio de acción (seg) & $\mathbf{n = 5 4}$ & $\mathbf{n = 4 6}$ & 0,000 \\
Duración clínica (min) & $120(90-150)$ & $180(120-270)$ & 0,000 \\
Índice de recuperación (min) & $79,5(67-105)$ & $44,5(33-63,5)$ & 0,799 \\
Duración del refuerzo (min) & $44(21,5-84)$ & $40(28,5-64)$ & 0,168 \\
\hline
\end{tabular}

TBW: por peso real; IBW: por peso ideal. 


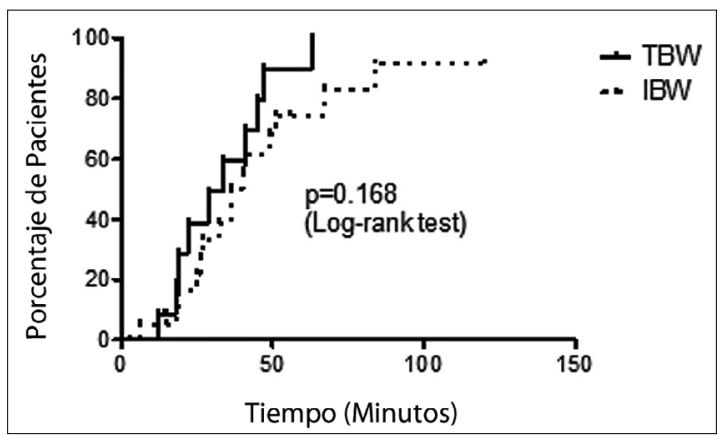

Figura 4. Duración de las dosis de refuerzo según dosificación por peso real o ideal.

pacientes dosificados por TBW. Esta diferencia no fue estadísticamente significativa; Odds Ratio $=2,2$ $(0,9-5,2), p=0,068$. La duración de un refuerzo de rocuronio hasta alcanzar una recuperación del $25 \%$ de $T_{1}$ no varió significativamente con la dosificación por TBW o por IBW (Figura 4).

\section{DISCUSIÓN}

Se ha recomendado que las dosis de los relajantes musculares en obesos sean calculada en base al peso ideal (IBW) debido a que al usar el peso corporal total (TBW) se teme una excesiva prolongación de la duración de acción ${ }^{14,17}$. Los estudios clínicos de duración de los bloqueadores neuromusculares en la literatura no han utilizado el análisis de sobrevida (análisis tiempo-evento), excepto Sluga $^{23}$, quien utilizó test de Log-rank. Esta es la metodología estadísticamente correcta puesto que los datos temporales tienden a ser asimétricos y lejos de ser normales ${ }^{20} \mathrm{y}$, por lo tanto, es incorrecto analizarlos con promedios, distribuciones estándar $\mathrm{y} t$ de student, que suponen un ajuste a una distribución normal.

El otro problema que se plantea al analizar datos temporales en condiciones clínicas es la presencia de censoring ${ }^{20,21}$; por ejemplo, si algunos pacientes nunca alcanzaron un $95 \%$ de bloqueo del primer estímulo del TOF ¿Cómo deben ser incorporados si en efecto no alcanzaron el grado de bloqueo que se fijó como meta? $\mathrm{O}$ bien, si otros pacientes debieron ser reforzados antes de que alcanzaran un $90 \%$ de recuperación del primer estímulo del TOF ¿Cómo estimaremos la duración de la droga en un estudio clínico? Esto se soluciona con el análisis de sobrevida que incorpora el censoring, es decir, aquellos pacientes que no alcanzan nunca el objetivo en estudio. El no incorporar a los pacientes con datos censurados introduce un sesgo de selección ${ }^{22}$. Este sesgo se produce de la siguiente manera: al tomar sólo los pacientes en que se pudo registrar hasta que alcanzaron un $95 \%$ de recuperación, estamos seleccionando a los pacientes en quienes el rocuronio tuvo una menor duración de acción y por lo tanto, la estimación de la duración del bloqueador neuromuscular será menor de la verdadera. Si consideramos los datos censurados (aquellos pacientes en los que no se alcanzó aún un $95 \%$ de recuperación) al menos sabremos que a un determinado tiempo aún no había recuperación completa, y por tanto, la duración del relajante se estimará más prolongada y más cercana entonces a la realidad, que es lo que podría haber ocurrido en los trabajos anteriores sobre el mismo tema ${ }^{24-27}$.

En nuestro estudio encontramos un significativo acortamiento del tiempo de inicio de acción al dosificar $0,6 \mathrm{mg} \cdot \mathrm{kg}^{-1}$ de rocuronio por peso real, permitiendo alcanzar un máximo de bloqueo (cero respuesta $T_{1}$ ) en 120 segundos, lo que ya había sido descrito por de Almeida en 200924. Por el contrario, se observó una prolongación de la duración clínica en dosis por peso real (TBW). Pühringer ${ }^{25}$ observó una prolongación significativa de la duración clínica al administrar $0,6 \mathrm{mg} \cdot \mathrm{kg}^{-1}$ de rocuronio en base al peso real a pacientes obesos en comparación con la misma dosis administrada a pacientes de peso subnormal, normal, y sobrepeso, sin diferencias en el tiempo de inicio ni en la recuperación espontánea o inducida; aquí obviamente la comparación es bastante sesgada puesto que existen otras diferencias entre los grupos en estudio que pudieran explicar estos resultados. El mismo autor ${ }^{26}$ no encontró diferencias en el tiempo de inicio, duración clínica y recuperación espontánea entre mujeres de peso normal y mujeres obesas. Leykin ${ }^{27}$ por el contrario, sí encontró una prolongación en la duración clínica: 22 minutos al dosificar por peso ideal y 55 minutos por peso real, pero no encontró diferencias en el tiempo de inicio. Cabe señalar que el tamaño muestral utilizado por este autor fue muy pequeño y probablemente no tuvo potencia suficiente para detectar diferencias. Meyhoff ${ }^{28}$ también encontró una significativa prolongación de la duración del rocuronio (definido como reaparición de la cuarta respuesta al TOF) al utilizar $0,6 \mathrm{mg} \cdot \mathrm{kg}^{-1}$ de peso ideal versus $0,6 \mathrm{mg} \cdot \mathrm{kg}^{-1}$ de peso ideal $+40 \%: 32$ minutos versus 42 minutos. Este autor tampoco encontró diferencias en el tiempo de inicio de acción.

Nuestros resultados mostraron una duración excesivamente prolongada en comparación a lo reportado en la literatura, incluso al dosificar por peso ideal. Esto puede ser atribuido a que los pacientes dosificados por peso real recibieron $1 \mathrm{mg} \cdot \mathrm{kg}^{-1} \mathrm{de}$ rocuronio (al ser calculado en base a su peso ideal), 
lo que se asemeja a la duración clínica reportada por Magorian ${ }^{29}$ y Wright $^{30}$ al utilizar dosis de 1,2 $\mathrm{mg} \cdot \mathrm{kg}^{-1}$. Nosotros no encontramos diferencia en el índice de recuperación al comparar a los pacientes que habían recibido dosis por peso real versus peso real. Esta independencia de la dosis del índice de recuperación en relación a la fórmula de cálculo de la dosis ha sido descrita en la literatura ${ }^{31}$. Es destacable que la duración del índice de recuperación en nuestros pacientes fue el doble de lo descrito por Leykin $^{26}$ y en general más prolongado que lo descrito en la literatura ${ }^{29-31}$, asemejándose al de los bloqueadores neuromusculares de larga duración ${ }^{31}$.

La causa de esto podría deberse a la gran incidencia de hígado graso que nuestros pacientes padecían (86\%), lo que podría jugar un papel en esta prolongación de la duración clínica, dado que el rocuronio es captado por el hígado y excretado en la bilis ${ }^{32}$ A esto se agrega el aumento de la presión intraabdominal, que de por sí está aumentada en obesos, y está además aumentada por el pneumoperitoneo, que se ha visto provoca algún grado de injuria hepática por hipoperfusión $^{33}$. Si bien el rocuronio es metabolizado solo parcialmente en el hígado, la excreción de drogas en la bilis requiere de la indemnidad del hepatocito ${ }^{34}$ y la esteatosis hepática se acompaña o progresa a inflamación ${ }^{35,36}$. Magorian ${ }^{37}$, comparó los efectos del rocuronio en pacientes con enfermedad hepática y normales, encontrando en pacientes con enfermedad hepática una duración clínica de $73 \pm$ 43 minutos, bastante similar a nuestros resultados en el grupo dosificado por TBW.

No hubo diferencias en la duración clínica de un primer refuerzo de rocuronio según la dosis por TBW o IBW, observándose una menor duración pero no estadísticamente significativa en el grupo
IBW. Dado que sólo un 32\% de los pacientes requirió refuerzo (13 pacientes en TBW y 19 pacientes en IBW), el estudio pudiera tener un poder insuficiente para obtener conclusiones. La duración de un primer refuerzo en nuestros pacientes fue similar a lo descrito por Servin ${ }^{38}$ en pacientes cirróticos: $30,9 \pm 1,7$ minutos (para una dosis de $0,15 \mathrm{mg} \cdot \mathrm{kg}^{-1}$, similar a nuestros refuerzos).

A diferencia de otros autores nosotros no usamos TIVA como método anestésico. No podemos descartar que la anestesia inhalatoria haya influenciado el curso del bloqueo neuromuscular ${ }^{39,40}$ y quizás ésta sea otra razón por la que nuestros tiempos de duración clínica con rocuronio hayan sido más prolongados que los observados en la mayoría de los estudios en los que se utilizó TIVA, que es el tipo de anestesia que menos potencia el bloqueo neuromuscular ${ }^{39}$, de manera que permite obtener una información más depurada. Sin embargo, creemos que es importante conocer los tiempos reales en las condiciones de trabajo habitual.

\section{CONCLUSIONES}

Al dosificar el rocuronio por peso real en pacientes obesos, se observó una prolongación de su duración clínica y un acortamiento de su tiempo de inicio de acción, en comparación con la dosificación en base al peso ideal.

Los pacientes obesos demostraron una mayor sensibilidad al rocuronio y por lo tanto, recomendamos administrarlo en base al peso ideal, reservando la dosificación en base al peso real para las situaciones que requieran de una inducción rápida.

\section{REFERENCIAS}

1. Yanovski SZ, Yanovski JA. Obesity prevalence in the United States-up, down, or sideways? N Engl J Med 2011; 364: 987-989.

2. Livingstone EH, Zylke JW. Progress in obesity research. Reasons for optimism. JAMA 2012; 308: 1162-1164.

3. Gobierno de Chile/Ministerio de Salud. Indicadores de obesidad en población chilena. Principales Resultados Encuesta Nacional de Salud 2010. Mayores de 15 años.

4. Guía Clínica Obesidad Mórbida. Serie guías Clínicas MINSAL. Santiago-Chile 2008.

5. Guzmán S, Manrique M, Raddatz
A, et al. Experiencia de 18 años de cirugía de obesidad en la Pontificia Universidad Católica de Chile. Rev Med Chile 2013; 141: 553-561.

6. Green B, Bulle SB. What is the best size descriptor to use for pharmacokinetic studies in the obese? $\mathrm{Br} \mathrm{J}$ Clin Pharmacol 2004; 58:119-133.

7. Lemmens HJM. Perioperative pharmacology in morbid obesity. Curr Opin Anaesthesiol 2010; 23: 485-491.

8. Schumann R, Jones SB, Cooper $\mathrm{B}$, et al. Update on best practice recommendations for anesthetic perioperative care and pain Management in weight loss surgery, 2004-2007. Obesity 2009; 17: 889894.
9. Adams JP, Murphy PG. Obesity in anaesthesia and critical care. Br J Anaesth 2000; 85: 91-108.

10. Dority J, Hassan ZU, Chau D. Anesthetic implications of obesity in the surgical patient. Clin $\mathrm{Col}$ Rectal Surg 2011; 24: 222-228.

11. Cullen A, Ferguson A. Perioperative Management of the severely obese patient: a selective pathophysiological review. Can J Anesth 2012; 59: 974-966.

12. Benumof JL. The new ASA OSA Guideline. The ASA refresher courses in anesthesiology 2007; 35: 1-13.

13. Ketherpal S, Han R, Tremper KK, et al. Incidence and predictors of difficult mask ventilation. Anesthe- 
siology 2006; 105: 885-891.

14. Álvarez A, Brodsky JB, Lemmens HJM, Morton JM (Eds.) 2010. Morbid Obesity. Peri-operative Management. Cambridge, UK: Cambridge University Press.

15. Rocuronium: the newest aminoesteroid neuromuscular blocking drug. Editorial II. Br J Anaesth 1996; 76 : 481-483.

16. Cheymol G. Effects of obesity on pharmacokinetics. Clin pharmacokinet 2000; 39: 215-231.

17. Ingrande J, Lemmens HJM. Dose adjustment of anaesthetics in the morbidly obese. Br J Anaesth 2010; 105: i16-i23.

18. Stata Release 10 (2007). Survival analysis and epidemiological tables, Lakeway Drive, College Station, Texas, pp. 208-219.

19. Lemmens HJM, Brodsky JB, Bernstein DP. Estimating ideal body weight-a new formula. Obesity Surgery $2005 ; 15: 1082-1083$.

20. Lee ET, Wang JW. (2003). Statistical Methods for Survival Data Analysis (3rd ed.) Hoboken New Jersey: John Wiley \& Sons Inc.

21. Kleinbaum, DG. (1996). Survival Analysis: a self-learning text. Gail M \& Singer B (eds.), New York, NY: Springer-Verlag.

22. Hernan MA, Robins JM. Causal Inference I. Chapman \& Hall CRC 2013.

23. Sluga M, Ummenhofer W, Studer W, et al. Rocuronium versus succinycholine for rapid secuence induction of anesthesia and endotracheal intubation: a prospective, randomized trial in emergent cases Anesth Analg 2005; 101: 13561361

24. De Almeida MC, Pedernairas SG, Chiaroni S, et al. Evaluation of tracheal intubation conditions in morbidly obese patients; a comparison of succinycholine and rocuro- nium. Rev Esp Anestesiol Reanim 2009; 56: 3-8.

25. Pühringer FK, Khuenl-Brady KS, Mitterschifthaler G. Rocuronium bromide: time-course of action in underweight, normal weight, overweight and obese patients. Eur J Anaesthesiol Suppl 1995; 11 : 107-110.

26. Puhringer FK, Keller C, Kleinsasser A, et al. Pharmacokinetics of rocuronium bromide in female obese patients. Eu J Anaesthesiol 1999; 16: 507-510.

27. Leykin Y, Pellis T, Lucca M, et al. The pharmacodynamics effects of rocuronium when dosed according to real body weight or ideal body weight in morbidly obese patients. Anesth Analg 2004; 99: 1086-1089.

28. Meyhoff C, Lund J, Jenstrum M, et al. Should dosing of rocuronium in obese patients be base on ideal or corrected body weight? Anesth Analg 2009; 107: 787-792.

29. Magorian T, Flannery KB, Miller $\mathrm{RD}$. Comparison of rocuronium, succinycholine and vecuronium for rapid secuence induction in adult patients. Anesthesiology 1993; 79: 913-918.

30. Wright PM, Caldwell JE, Miller RD. Onset and duration of rocuronium and succinycholine at the adductor pollicis and laryngeal adductor muscles in anesthetized humans. Anesthesiology 1994; 81: 1110-1115.

31. Barash PG, Cullen BC, Stoelting RK, et al. Neuromuscular blocking agents In: Brown B, Dernosky NT, Crater P, editors. Clinical Anesthesia. Philadelphia PA: Lippincott Williams \& Wilkins; 2009; p. 498530.

32. Proost JH, Eriksson LI, Mirakhur RK, et al. Uinary, biliary and fecal excretion of rocuronium in humans. BJA 2000; 85: 717-723.
33. Nguyen N, Wolfe B. The physiologic effects of pneumoperitoneum in the morbidly obese. Ann Surg 2005; 241: 219-226.

34. Mizuno N, Niwa T, Yotsumoto Y, et al. Impact of drug transport studies on drug transport and development. Pharmacol Rev 2003; 55: 425-461.

35. Sattar N, Preiss D. Non-alcoholic fatty liver disease: an overview of prevalence, diagnosis, patogénesis and treatment considerations. Clinical Science 2008; 115: 141-150.

36. Chalasani N, Younossi Z, Lavine JE, et al. The diagnosis and management of Non-Alcoholic Fatty Liver Disease: Practice Guideline by the American Association for the study of liver diseases, American College of Gastroenterology and the American Gastroenterological Association. Hepatology 2012; 55: 2005-2023.

37. Magorian T, Wood P, Caldwell J, et al. The pharmacokinetics and neuromuscular effects of rocuronium bromide in patients with liver disease. Anesth Analg 1995; 80754-759.

38. Servin FS, Lavaut E, Kleef U, et al. Repeated doses of rocuronium bromide administered to cirrhotic and control patients receiving isoflurane. Anesthesiology 1996; 84: 1092-1100.

39. Wulf H, Kahl M, Ledowski T. Augmentation of the neuromuscular blocking effects of cisatracurium during desflurane, sevoflurane, isoflurane or total i.v. anaesthesia. Br J Anaesth 1998; 80: 308-312.

40. Paul M, Fokt RM, Kindler CH, et al. Characterization of the interactions between volatile anesthetics and neuromuscular blockers at the muscle nicotinic acetylcholine receptor. Anesth Analg 2002; 59: 362-367.

\footnotetext{
Correspondencia a:

Dagoberto Ojeda D.

eojedadinamarca@gmail.com
} 